\title{
SEMI-ANALYTICAL SOLUTION FOR RADIATION OF A HOLLOW SPHERE WITH AN OPENING HOLE IN FINITE DEPTH
}

\section{Man-sheng Dong}

State Key Laboratory of Ocean Engineering, Shanghai Jiaotong University,Shanghai, P.R.C.School of Transportation Engineering, Hefei University of Technology, Hefei,P.R.C

Guo-ping Miao

State Key Laboratory of Ocean Engineering, Shanghai Jiaotong University, Shanghai, P.R.C., gpmiao@sjtu.edu.cn

Ju Fan

State Key Laboratory of Ocean Engineering, Shanghai Jiaotong University, Shanghai, P.R.C.

Ren-chuan Zhu

Follow this and additional works at: https://jmstt.ntou.edu.tw/journal

Part of the Ocean Engineering Commons

\section{Recommended Citation}

Dong, Man-sheng; Miao, Guo-ping; Fan, Ju; and Zhu, Ren-chuan (2012) "SEMI-ANALYTICAL SOLUTION FOR RADIATION OF A HOLLOW SPHERE WITH AN OPENING HOLE IN FINITE DEPTH," Journal of Marine Science and Technology. Vol. 20: Iss. 1, Article 10.

DOI: $10.51400 / 2709-6998.2425$

Available at: https://jmstt.ntou.edu.tw/journal/vol20/iss1/10

This Research Article is brought to you for free and open access by Journal of Marine Science and Technology. It has been accepted for inclusion in Journal of Marine Science and Technology by an authorized editor of Journal of Marine Science and Technology. 


\title{
SEMI-ANALYTICAL SOLUTION FOR RADIATION OF A HOLLOW SPHERE WITH AN OPENING HOLE IN FINITE DEPTH
}

\author{
Man-sheng Dong ${ }^{1,2}$, Guo-ping Miao ${ }^{1}, \mathrm{Ju}_{\mathrm{Fan}}{ }^{1}$, and Ren-chuan Zhu ${ }^{1}$
}

Key words: radiation, submerged sphere, multipole potential.

\begin{abstract}
An investigation is conducted on radiation of a submerged hollow sphere with an opening hole in finite water depth in this article. Based on the linear theory, the method of multipole expansions is used to obtain the fluid velocity potential in the form of double series of the associated Legendre functions with the unknown coefficients of an infinite set. In terms of the body surface boundary condition and the matching condition between the inner and outer flows at the hole, the complex matrix equations for the coefficients of the series are established. The infinite sets of matrix equations are solved by truncating the series at a finite number. Subsequently, the added mass and radiation damping, associated with the periodic heave motion of a submerged sphere, are evaluated numerically.
\end{abstract}

\section{INTRODUCTION}

It is always a widely concerned issue in the offshore and ocean industries that radiation wave is caused by motion of ocean engineering structure. There has been a mass of research work dedicated to solve the radiation problem.

The method of multipole expansions is based on a oneparameter family of solutions of the boundary value problem $[1,6,8,9]$, which consists of two terms a singular solution of the Laplace equation and integral component. Thorne [12] introduced the method of multipole expansions for both spherical and cylindrical structures in finite and infinite depths. Wang [13], based on the multipole expansions presented by Thorne, investigated the radiation and diffraction problems for submerged spherical vehicles, and discussed their free motions and different metacentric heights in infinite depth. Lin-

Paper submitted 05/28/10; revised 09/06/10; accepted 10/11/10. Author for correspondence: Guo-ping Miao (e-mail: gpmiao@sjtu.edu.cn).

${ }^{1}$ State Key Laboratory of Ocean Engineering, Shanghai Jiaotong University, Shanghai, P.R.C.

${ }^{2}$ School of Transportation Engineering, Hefei University of Technology, Hefei, P.R.C. ton [6] worked on radiation and diffraction of water waves by a submerged sphere in finite depth using the multipole method, and presented the method to predict the free surface elevation in the vicinity of the sphere.

The boundary element method and finite element method can be used to solve radiation problem. Teng et al. [11] described some higher-order boundary element methods and presents a novel integral equation for the calculation of the wave diffraction and radiation problem. Bai et al. [2] used a higher-order boundary element method to simulate numerically nonlinear waves radiated by a forced oscillating vertical circular cylinder and solve the mixed boundary value problem based on an Eulerian description. Wang et al. [14] investigated numerically viscous flow past a rotating sphere by solving the three-dimensional Navier-Stokes equations using the finite element method.

Panel method is a kind of approach to analyze radiation problem. Qiu et al. [7] developed a panel-free method (PFM) to solve the radiation problem of a floating body in the time domain. The velocity potential due to a non-impulsive velocity is obtained by solving the boundary integral equation in terms of source strength distribution. Datta et al. [3] developed an open uniform B-spline-based panel method for solution of potential flow problems. The method was applied for the radiation problem in unbounded fluid and diffraction problem formulated based on the transient Green's function. Gao et al. [4, 5] developed a high-order Rankine panel method based on Non-Uniform Rational B-spline (NURBS) to solve the three-dimensional radiation and diffraction problems with forward speed.

Besides foregoing methods, there are other methods for this problem. For instance, Siddorn et al. [10] solved the radiation and diffraction of linear waves by an array of and a square array of truncated cylinders in the frequency domain by an exact algebraic method. Zheng et al. [15] analyzed the radiation and the diffraction of wave by an infinitely long floating rectangular structure submerged in water of finite depth with leeward boundary being a vertical wall by the eigenfunction expansion matching method.

For some reasons, in some specific areas the spherical structure may not be a whole sphere, but with an opening hole. According to the existing literature, the authors have not found 


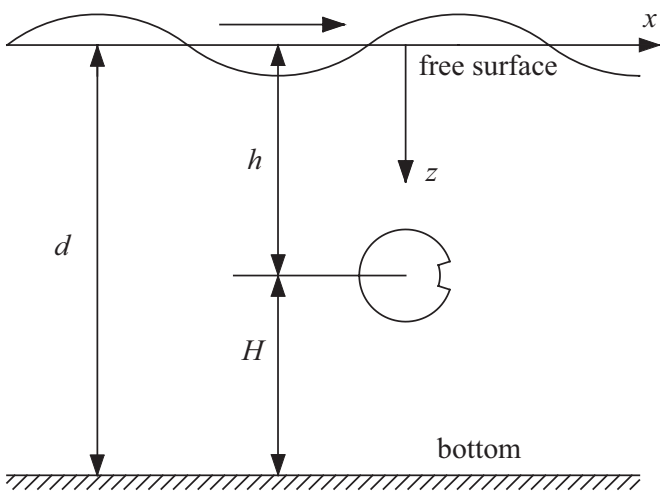

Fig. 1. Sketch of geometry for the problem.

an investigation on radiation of a sphere with an opening hole. In this paper, an analytical procedure for radiation of a submerged sphere with an opening hole is presented.

\section{FORMULATION OF THE PROBLEM}

\section{Elementary Formula}

It is assumed that the fluid is homogeneous, inviscid and incompressible and the fluid motion is irrotational. A surface wave with the frequency $\sigma$ and small amplitude $A$ is applied on a hollow sphere of the radius a submerged in water of the finite depth $d$. The wave is parallel to the $x$-axis at the time of the incidence on the sphere and is propagating along the positive $x$-direction.

Two sets of coordinate systems are taken. One is a righthanded Cartesian coordinate system $(x, y, z)$, in which the $x y$ plane coincides with the undisturbed free surface and the $z$-axis is taken vertically downwards from the free surface. The geometric centre of the sphere is located at the point $(0,0, h)$. The other set is the spherical coordinate system $(r, \theta, \psi)$ with the origin at the geometric centre of the sphere. Fig. 1 shows the brief sketch of geometry for the problem.

The relationship between the coordinate systems is given by $z-h=r \cos \theta, x=r \sin \theta \cos \psi, y=r \sin \theta \sin \psi$, such that

$$
\begin{gathered}
R=r \sin \theta=\sqrt{x^{2}+y^{2}} \\
r=\sqrt{R^{2}+(z-h)^{2}} \\
\tan \theta=\frac{R}{z-h}, \quad 0 \leq \theta \leq \pi
\end{gathered}
$$

and

$$
\tan \psi=\frac{y}{x}, \quad-\pi \leq \psi \leq \pi
$$

It is assumed that a rectangle aperture is situated at the sphere in the direction of wave propagation. The longitudinal range of angle is $-\psi_{\mathrm{h}} \leq \psi \leq \psi_{\mathrm{h}}$, and the latitudinal range of angle is $\pi / 2-\theta_{\mathrm{h}} \leq \theta \leq \pi / 2+\theta_{\mathrm{h}}$.

For an incompressible and inviscid fluid, and for the small amplitude wave theory with irrotational motion, a velocity potential $\Phi(r, \theta, \psi, t)$ can describe the fluid motion, which is written as:

$$
\Phi(r, \theta, \psi, t)=\operatorname{Re}\left[\phi(r, \theta, \psi) e^{\mathrm{i} \sigma t}\right]
$$

where Re stands for the real part, $\phi$ is a time independent potential and $\sigma$ is the frequency of oscillation.

\section{The Velocity Potential Outside the Sphere}

To obtain the velocity potential $\phi=\phi_{\mathrm{R}}$ for radiation, the following boundary value problem is to be solved

$$
\nabla^{2} \phi=0
$$

$$
\frac{\partial \phi}{\partial z}+K \phi=0, \quad \text { on } z=0
$$

$$
\frac{\partial \phi}{\partial z}=0, \quad \text { on } z=d
$$

$$
\sqrt{R}\left(\frac{\partial}{\partial R}-\mathrm{i} k_{0}\right) \phi_{\mathrm{R}}=0, \quad \text { at infinity }
$$

$$
\frac{\partial \phi_{\mathrm{R}}}{\partial r}=V_{\mathrm{r}}, \quad \text { on } r=a \text { except the opening hole }
$$

where $V_{\mathrm{r}}$ is normal velocity with the direction of the normal defined out of the fluid, $K=\sigma^{2} / g$ and $k_{0}$ is the finite depth wave-number defined by the dispersion relation

$$
k_{0} \sinh k_{0} d-K \cosh k_{0} d=0
$$

The radial velocity $V_{\mathrm{r}}$ of a sphere surface is defined by oscillation mode. Oscillation modes of the sphere are mainly heave and surge. Since procedures of dealing with two problems are similar, we are focused on heave motion. Since motion amplitude is small in linear theory, the boundary condition of body face is met on the average body surface.

\section{The Velocity Potential Outside the Sphere}

The velocity potential inside the sphere is denoted by $\phi_{\text {inner. }}$. Governing equation reads

$$
\nabla^{2} \phi_{\text {inner }}=0
$$

$$
\frac{\partial \phi_{\text {inner }}}{\partial r}=V_{\mathrm{r}} \text {, on } r=a \text { except the opening hole }
$$


The matching condition of the outside flow field and the inner flow field reads

$$
\frac{\partial \phi_{\mathrm{R}}}{\partial r}=\frac{\partial \phi_{\text {inner }}}{\partial r}, \text { on } r=a,-\pi \leq \psi \leq \pi, 0 \leq \theta \leq \pi
$$

Further they can be written as

$$
\frac{\partial \phi_{\mathrm{R}}}{\partial r}=\frac{\partial \phi_{\text {inner }}}{\partial r}=V_{\mathrm{r}}, \text { on } r=a,\left\{\begin{array}{c}
\left(-\pi,-\psi_{\mathrm{h}}\right) \text { and }\left(\psi_{\mathrm{h}}, \pi\right) \\
\left(0, \frac{\pi}{2}-\theta_{\mathrm{h}}\right) \text { and }\left(\frac{\pi}{2}+\theta_{\mathrm{h}}, \pi\right)
\end{array}\right.
$$

$\phi_{\mathrm{R}}=\phi_{\text {inner }}$, on $r=a,-\psi_{\mathrm{h}} \leq \psi \leq \psi_{\mathrm{h}}, \frac{\pi}{2}-\theta_{\mathrm{h}} \leq \theta \leq \frac{\pi}{2}+\theta_{\mathrm{h}}$

\section{MULTIPOLE EXPANSIONS}

\section{The Inner Potential}

By the method of separation of variables, the inner velocity potential $\phi_{\text {inner }}$ can be expressed as $\phi_{\text {ineer }}=R(r) \Theta(\theta) \Psi(\psi)$.

Using separation of variables for Eq. (6) we have

$$
\begin{gathered}
\frac{\mathrm{d}^{2} \Psi}{\mathrm{d} \psi^{2}}+m^{2} \Psi=0 \\
r^{2} \frac{\mathrm{d}^{2} R}{\mathrm{~d} r^{2}}+2 r \frac{\mathrm{d} R}{\mathrm{~d} r}-l(l+1) R=0 \\
\frac{1}{\sin \theta} \frac{\mathrm{d}}{\mathrm{d} \theta}\left(\sin \theta \frac{\mathrm{d} \Theta}{\mathrm{d} \theta}\right)+\left(\mu-\frac{m^{2}}{\sin ^{2} \theta}\right) \Theta=0
\end{gathered}
$$

where $\mu=l(l+1), 0 \leq \theta \leq \pi$.

The general solution to Eq. (17) is $\Psi(\psi)=\cos m \psi$, where $m$ is an integer. The general solution to Eq. (18) is $R(r)=A r^{l}+$ $B r^{-l-1}$, where $l$ is an integer. For $R(r), r^{-l-1}$ is close to infinity when $r \rightarrow 0$, which goes against physical phenomenon. So $r^{-l-1}$ should be removed, and $r^{l}$ is held.

The general solution to Eq. (19) is the associated Legendre function of $l$ degree and $m$ order

$$
P_{l}^{m}(x)=\left(1-x^{2}\right)^{m / 2} \frac{\mathrm{d}^{m}}{\mathrm{~d} x^{m}} P_{l}(x), \quad 0 \leq m \leq l
$$

where $P_{l}(x)$ is the Legendre polynomial of degree $l, x=\cos \theta$.

It is known that different levels and orders of associated Legendre functions have the orthogonal properties.

$$
\int_{0}^{\pi} P_{l}^{m}(\cos \theta) P_{k}^{m}(\cos \theta) \sin \theta \mathrm{d} \theta=\frac{(l+m) !}{(l-m) ! 2 l+1} \frac{2}{l k}
$$

Thus the inner velocity potential $\phi_{\text {inner }}$ can be expressed by

$$
\begin{gathered}
\phi_{\text {inner }}=\sum_{m=0}^{\infty} \phi_{m}^{\mathrm{inner}}(r, \theta) \cos m \psi \\
\phi_{m}^{\mathrm{inner}}(r, \theta)=\sum_{n=m}^{\infty} B_{n}^{m} r^{n} P_{n}^{m}(\cos \theta)
\end{gathered}
$$

We take the first derivative of $\phi_{\text {ineer }}$ with respect to $r$ and have

$$
\frac{\partial \phi_{m}^{\mathrm{inner}}}{\partial r}=\sum_{n=m}^{\infty} B_{n}^{m} n r^{n-1} P_{n}^{m}(\cos \theta)
$$

If $\mathrm{m}$ is truncated to $M$, the number of unknown quantities $B_{n}^{m}$ is $(M+1)(M+2) / 2$.

\section{The Outer Potential}

The outer velocity potential $\phi_{\mathrm{R}}$ reads

$$
\phi_{\mathrm{R}}(r, \theta, \psi)=\sum_{m=0}^{\infty} \phi_{m}^{\mathrm{R}}(r, \theta) \cos m \psi
$$

where the $\psi$-independent potential is

$$
\phi_{m}^{\mathrm{R}}(r, \theta)=\sum_{n=m}^{\infty} a^{n+2} A_{n}^{m} G_{n}^{m}(r, \theta)
$$

Here $A_{n}^{m}$ are the unknown coefficients and $G_{n}^{m}(r, \theta)$ are the multipole potentials. The function $G_{n}^{m}(r, \theta) \cos m \psi$ satisfies the Laplace equation, the free surface and bottom boundary conditions and the radiation boundary condition except the body surface boundary condition.

The function $G_{n}^{m}(r, \theta)$ can be expressed as [8]

$$
\begin{aligned}
G_{n}^{m}= & \frac{P_{n}^{m}(\cos \theta)}{r^{n+1}}+\frac{P_{n}^{m}(\cos \alpha)}{r_{1}^{n+1}} \\
& +\frac{1}{(n-m) !} \int_{0}^{\infty} \frac{(K+k)\left[e^{-k(d+H)}+(-1)^{n+m} e^{-k h}\right]}{(k \sinh k d-K \cosh k d)} \\
& \times k^{n} \cosh k(z-d) J_{m}(k R) \mathrm{d} k
\end{aligned}
$$

where $r_{1}$ and $\alpha$ are defined as

$$
r_{1}=\sqrt{R^{2}+(d+H-z)^{2}}, \quad \tan \alpha=\frac{R}{(d+H-z)} .
$$

The line of integration in Eq. (27) passes under the singular point of the integrand at $k=k_{0}$. 
The second and third terms in Eq. (27) can be expanded into a series of the associated Legendre functions by

$$
\frac{P_{n}^{m}(\cos \alpha)}{r_{1}^{n+1}}=\sum_{s=0}^{\infty} B_{n s}^{m}\left(\frac{r}{2 H}\right)^{s+m} P_{s+m}^{m}(\cos \theta)
$$

and

$$
\begin{aligned}
& \frac{1}{(n-m) !} \int_{0}^{\infty} \frac{(K+k)\left[e^{-k(d+H)}+(-1)^{n+m} e^{-k h}\right]}{(k \sinh k d-K \cosh k d)} k^{n} \cosh k(z-d) J_{m}(k R) \mathrm{d} k \\
& =\sum_{s=0}^{\infty} C_{n s}^{m}\left(\frac{r}{2 H}\right)^{s+m} P_{s+m}^{m}(\cos \theta)
\end{aligned}
$$

where $B_{n s}^{m}$ and $C_{n s}^{m}$ are defined by

$$
B_{n s}^{m}=\frac{1}{(2 H)^{n+1}} \frac{(n+m+s) !}{(n-m) !(s+2 m) !}
$$

and

$$
\begin{aligned}
C_{n s}^{m}= & \frac{(2 H)^{s+m}}{(n-m) !(s+2 m) !} \\
& \int_{0}^{\infty} \frac{(K+k)\left[e^{-k(d+H)}+(-1)^{n+m} e^{-k h}\right]}{(k \sinh k d-K \cosh k d)} k^{n+m+s} U_{s}(k H) \mathrm{d} k
\end{aligned}
$$

where $U_{s}(\xi)$ is defined by

$$
U_{s}(\xi)= \begin{cases}\cosh \xi, & s=0,2,4, \cdots \\ -\sinh \xi, & s=1,3,5, \cdots\end{cases}
$$

Hence the multipole potential $G_{n}^{m}(r, \theta)$ can finally be written as:

$$
G_{n}^{m}(r, \theta)=\frac{P_{n}^{m}(\cos \theta)}{r^{n+1}}+\sum_{s=0}^{\infty}\left(B_{n s}^{m}+C_{n s}^{m}\right)\left(\frac{r}{2 H}\right)^{s+m} P_{s+m}^{m}(\cos \theta)
$$

If $m$ is truncated to $M$, the number of unknown quantities $A_{n}^{m}$ is $(M+1)(M+2) / 2$. We notice that the problem under consideration contains $(M+1)(M+2)$ unknown coefficients. Hence we have to establish $(M+1)(M+2)$ equations to determine these coefficients. Next we present the establishment of these equations.

\section{Matching the Inner and Outer Flow Fields}

There are two matching conditions for the hollow sphere surface, including the opening hole. In other words, the ve- locity potentials satisfy both the body surface condition and the opening hole condition, i.e. Eqs. (15) and (16).

On the inner wall surface of the hollow sphere, both sides of Eq. (13) are equal to $V_{\mathrm{r}}$, which are satisfied by functions introduced below. At the opening hole, the normal derivatives of the inner and outer field potentials are equal.

We suppose that the structure oscillates harmonically. The velocity is denoted by $V=V_{i} e^{i \sigma t}, i=0,1$, where $\sigma$ is oscillating frequency of the structure. Its harmonic oscillation may be depicted by two modes heave and sway, which are denoted by suffix 0 and 1 , respectively. $V_{i}$ may be token by unity velocity.

Thus the radial velocity component $V_{0}^{\mathrm{r}}$ of heave reads

$$
V_{0}^{\mathrm{r}}=\cos \theta
$$

The radial velocity component $V_{1}^{\mathrm{r}}$ of sway reads

$$
V_{1}^{\mathrm{r}}=\sin \theta \cos \psi
$$

We note that $P_{1}^{0}(\cos \theta)=\cos \theta, P_{1}^{1}(\cos \theta)=\sin \theta$. Hence $V_{j}^{\mathrm{r}}$ can be denoted by the identity below

$$
V_{j}^{\mathrm{r}}=P_{1}^{j}(\cos \theta) \cos j \psi, \quad j=0,1
$$

where $j$ is a definite number, not iterative suffix. $j=0,1$ means heave and sway modes, respectively.

Further we definite a function following

$$
\begin{aligned}
& \tilde{G}=\left\{\begin{array}{c}
\phi_{\mathrm{R}}-\phi_{\text {inner }} \\
\frac{\partial \phi_{\text {inner }}}{\partial r}-V_{j}^{\mathrm{r}}
\end{array}\right\}=0, \text { on } \\
& r=a,\left\{\begin{array}{c}
-\psi_{\mathrm{h}} \leq \psi \leq \psi_{\mathrm{h}}, \quad \frac{\pi}{2}-\theta_{\mathrm{h}} \leq \theta \leq \frac{\pi}{2}+\theta_{\mathrm{h}} \\
\psi\left(\begin{array}{c}
\psi:\left(\psi_{\mathrm{h}}, 2 \pi-\psi_{\mathrm{h}}\right), \quad \theta:\left(\frac{\pi}{2}-\theta_{\mathrm{h}}, \frac{\pi}{2}+\theta_{\mathrm{h}}\right) \\
\psi:(0,2 \pi), \quad \theta:\left(0, \frac{\pi}{2}-\theta_{\mathrm{h}}\right) \text { and }\left(\frac{\pi}{2}+\theta_{\mathrm{h}}, \pi\right)
\end{array}\right.
\end{array}\right.
\end{aligned}
$$

Hence Eq. (37) satisfy the matching conditions, which can determine uniquely unknown coefficients in formal solutions. According to Eq. (14), we have

$$
\left.\sum_{n=m}^{\infty} a^{n+2} A_{n}^{m} \frac{\partial G_{n}^{m}}{\partial r}\right|_{r=a}=\left.\sum_{n=m}^{\infty} n B_{n}^{m} r^{n-1} P_{n}^{m}(\cos \theta)\right|_{r=a}
$$

In terms of Eq. (33), we can obtain

$$
\sum_{n=m}^{\infty} A_{n}^{m} E_{n s}^{m}=B_{s}^{m} Q_{s}^{m}, s=m, m+1, \cdots ; m=0,1, \cdots,
$$


where

$$
\begin{gathered}
E_{n s}^{m}=-(n+1) \delta_{n s}+D_{n, s-m}^{m} \\
D_{n s}^{m}=a^{n+1}(s+m)\left(B_{n s}^{m}+C_{n s}^{m}\right)\left(\frac{a}{2 H}\right)^{s+m} \\
Q_{s}^{m}=s a^{s-1}
\end{gathered}
$$

And $\delta_{n s}$ is the Kronecker delta function.

As above, if $m$ is truncated to $M$, there are $(M+1)(M+2) / 2$ linear equations with unknown coefficients $A_{n}^{m}$ and $B_{n}^{m}$.

We discompose the sphere surface into three sections $(0$, $\left.\pi / 2-\theta_{\mathrm{h}}\right),\left(\pi / 2-\theta_{\mathrm{h}}, \pi / 2+\theta_{\mathrm{h}}\right)$ and $\left(\pi / 2+\theta_{\mathrm{h}}, \pi\right)$ in latitude direction. The opening hole is at $\left(\pi / 2-\theta_{\mathrm{h}}, \pi / 2+\theta_{\mathrm{h}}\right)$. The sphere surface boundary of latitude degree $\left(0, \pi / 2-\theta_{\mathrm{h}}\right)$ and $\left(\pi / 2+\theta_{\mathrm{h}}, \pi\right)$ is wall boundary. According to Eq. (37), we have

$$
\begin{gathered}
\frac{\partial \phi_{\text {inner }}}{\partial r}=P_{1}^{j}(\cos \theta) \cos j \psi, \text { on } r=a, \\
\psi:(0,2 \pi), \\
\theta:\left(0, \frac{\pi}{2}-\theta_{\mathrm{h}}\right) \text { and }\left(\frac{\pi}{2}+\theta_{\mathrm{h}}, \pi\right)
\end{gathered}
$$

and

$$
\begin{aligned}
& \left.\begin{array}{c}
\phi_{\mathrm{R}}-\phi_{\text {inner }} \\
\frac{\partial \phi_{\text {inner }}}{\partial r}-P_{1}^{j}(\cos \theta) \cos j \psi
\end{array}\right\}=0 \text {, on } r=a \text {, } \\
& \theta:\left(\frac{\pi}{2}-\theta_{\mathrm{h}}, \frac{\pi}{2}+\theta_{\mathrm{h}}\right),\left\{\begin{array}{c}
\psi:\left(-\psi_{\mathrm{h}}, \psi_{\mathrm{h}}\right) \\
\psi:\left(\psi_{\mathrm{h}}, 2 \pi-\psi_{\mathrm{h}}\right)
\end{array}\right.
\end{aligned}
$$

Both sides of Eqs. (43) and (44) are multiplied by $\cos k \psi$, respectively, and integrated from 0 to $2 \pi$. According to the orthogonality properties of $m \psi$ cosine functions at the extent $(0,2 \pi)$, noticing Eqs. (22), (24), (27), (28), (30), (31) and (37) we have

$$
\begin{aligned}
& \sum_{n=k}^{\infty} B_{n}^{k} n a^{n-1} P_{n}^{k}(\cos \theta)-P_{1}^{k}(\cos \theta) \delta_{k j}=0, k=0,1, \cdots, \\
& \theta:\left(0, \frac{\pi}{2}-\theta_{h}\right) \text { and }\left(\frac{\pi}{2}+\theta_{h}, \pi\right)
\end{aligned}
$$

and

$$
\begin{aligned}
& \sum_{m=0}^{\infty} C 1_{m k}\left\{\sum _ { n = m } ^ { \infty } a ^ { n + 2 } A _ { n } ^ { m } \left[\frac{P_{n}^{m}(\cos \theta)}{a^{n+1}}\right.\right. \\
& \left.\left.+\sum_{s=0}^{\infty}\left(B_{n s}^{m}+C_{n s}^{m}\right)\left(\frac{a}{2 H}\right)^{s+m} P_{s+m}^{m}(\cos \theta)\right]-\sum_{n=m}^{\infty} B_{n}^{m} a^{n} P_{n}^{m}(\cos \theta)\right\}
\end{aligned}
$$

$$
\begin{aligned}
& +\sum_{m=0}^{\infty} C 2_{m k} \sum_{n=m}^{\infty} B_{n}^{m} n a^{n-1} P_{n}^{m}(\cos \theta)-C 2_{j k} P_{1}^{j}(\cos \theta)=0, \\
& \theta:\left(\frac{\pi}{2}-\theta_{h}, \frac{\pi}{2}+\theta_{h}\right), k=0,1, \cdots
\end{aligned}
$$

where

$$
C 1_{m k}=\int_{-\psi_{h}}^{\psi_{h}} \cos m \psi \cos k \psi \mathrm{d} \psi, C 2_{m k}=\int_{\psi_{h}}^{2 \pi-\psi_{h}} \cos m \psi \cos k \psi \mathrm{d} \psi
$$

In terms of the orthogonality of associated Legendre polynomials in $\theta$ interval of 0 to $\pi$, Eqs. (45) and (46) are multiplied by $P_{l}^{k}(\cos \theta) \sin \theta$ and integrated for $\theta$ over the interval $[0, \pi]$. Considering the definition extent of these equations, we define

$$
\begin{gathered}
I H_{l n}^{k m}=\int_{\frac{\pi}{2}+\theta_{h}}^{\pi} P_{l}^{k}(\cos \theta) P_{n}^{m}(\cos \theta) \sin \theta \mathrm{d} \theta \\
I M_{l n}^{k m}=\int_{\frac{\pi}{2}-\theta_{h}}^{\frac{\pi}{2}+\theta_{h}} P_{l}^{k}(\cos \theta) P_{n}^{m}(\cos \theta) \sin \theta \mathrm{d} \theta \\
I L_{l n}^{k m}=\int_{0}^{\frac{\pi}{2}-\theta_{h}} P_{l}^{k}(\cos \theta) P_{n}^{m}(\cos \theta) \sin \theta \mathrm{d} \theta
\end{gathered}
$$

We have easily

$$
\begin{aligned}
& \sum_{n=k}^{\infty} B_{n}^{k} n a^{n-1}\left(I L_{l n}^{k k}+I H_{l n}^{k k}\right)-\left(I L_{l 1}^{k k}+I H_{l 1}^{k k}\right) \delta_{k j} \\
& +\sum_{m=0}^{\infty} C 1_{m k}\left\{\sum_{n=m}^{\infty} a^{n+2} A_{n}^{m}\left[\frac{I M_{l n}^{k m}}{a^{n+1}}+\sum_{s=0}^{\infty}\left(B_{n s}^{m}+C_{n s}^{m}\right)\left(\frac{a}{2 H}\right)^{s+m} I M_{l, s+m}^{k m}\right]\right. \\
& \left.-\sum_{n=m}^{\infty} B_{n}^{m} a^{n} I M_{l n}^{k m}\right\}+\sum_{m=0}^{\infty} C 2_{m k} \sum_{n=m}^{\infty} B_{n}^{m} n a^{n-1} I M_{l n}^{k m} \\
& -C 2_{j k} I M_{l 1}^{k j}=0, \quad k=0,1, \cdots, l=k, k+1, \cdots .
\end{aligned}
$$

Eq. (50) is similar to Eq. (38) in unknowns. If $k$ is truncated to $M$, there are $(M+1)(M+2) / 2$ linear equations with unknown coefficients $A_{n}^{m}$ and $B_{n}^{m}$. Thus there are $(M+1)(M+2)$ linear equations with unknown coefficients $A_{n}^{m}$ and $B_{n}^{m}$ for Eqs. (38) and (50). Obviously, the number of unknown coefficients is also $(M+1)(M+2)$. Through solving these equations we can determine unknown $A_{n}^{m}$ and $B_{n}^{m}$ and accordingly obtain the inner and outer velocity potentials. 


\section{THE ADDED MASS AND RADIATION DAMPING}

The total exciting forces on the sphere by the radiation potential can be calculated by integrating the total pressure $P$ over the body surface $\Omega$

$$
\mathbf{F}_{\mathrm{R}}=\iint_{\Omega} P \mathbf{n} d S
$$

Here $\mathbf{n}$ is the normal vector out of the body surface having the components

$$
\mathbf{n}=\left(n_{x}, n_{y}, n_{z}\right)
$$

where $n_{x}=\sin \theta \cos \psi, n_{y}=\sin \theta \sin \psi$, and $n_{z}=\cos \theta$.

Let the motion of the sphere be described by $\operatorname{Re}\left[\left(\xi_{0}, \xi_{1}\right) e^{i \sigma t}\right]$ for heave and surge motion, respectively. We then define $\phi_{\mathrm{R}}^{j}$ by $\Phi=\operatorname{Re}\left[\mathbf{i} \omega \xi_{j} \phi_{\mathrm{R}}^{j} \mathrm{e}^{\mathrm{i} \sigma t}\right](j=0,1)$, with $\phi_{\mathrm{R}}^{j}$ representing the radiation potential due to a velocity of unit amplitude in the jth mode. As the radiation potential $\phi_{\mathrm{R}}^{j}$ is solved, the hydrodynamic forces can be evaluated though Eq. (51). Accordingly if we write

$$
\left(\mathbf{F}_{\mathrm{R}}\right)_{i, j}=\omega^{2} A_{i, j}-\mathbf{i} \omega B_{i, j}=\omega^{2} \rho \iint_{\Omega} \phi_{\mathrm{R}}^{j} n_{i} \mathrm{~d} s
$$

where $A_{i, j}$ and $B_{i, j}$ are known as the added mass and radiation damping, respectively. The subscript $j$ denotes the mode of motions, where $i=x, y, z, j=0$ for heave and $j=1$ for surge.

The rapid convergence of the infinite series solutions in terms of the associated Legendre functions has been found with respect to radiation parameter $K a$. So we can solve the infinite equations by truncating the series at a finite number $n=N$. Rahman [8] indicated that $N=4$ is sufficient to obtain accurate enough results. In this article $N=4$ was taken to make numerical simulation.

Numerical analysis was conducted to observe the relationship between the added mass and radiation damping and angles of opening hole. Main parameters of numerical simulation are as follows: $\theta_{\mathrm{h}}=\psi_{\mathrm{h}}=\theta, j=0, a=1$, the submergence parameter $h / a=2$, depth parameter $d / a=6$, and opening hole angles from 0.2 to 0.4 at interval 0.1 . Since non-dimensionalized added mass and radiation damping coefficients are slightly different for various opening angles, we take directly added mass and radiation damping to observe the influence of opening angle. Numerical results are demonstrated by the following Figs. 2 and 3.

Fig. 2 and Fig. 3 show heave added mass and radiation damping in the condition of various opening angles, respectively. We can see that added mass tends to increase with opening angles getting greater. The larger opening hole angle is, the more it increases. When $\theta_{\mathrm{h}}=\psi_{\mathrm{h}}=\theta=0.4$, it go up by close 10 per cent. But heave radiation damping is visible

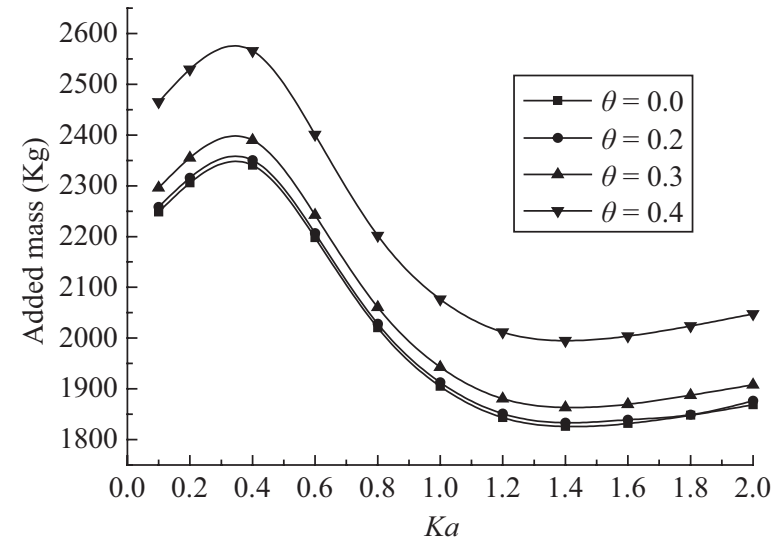

Fig. 2. Heave added mass for a submerged sphere with an opening hole.

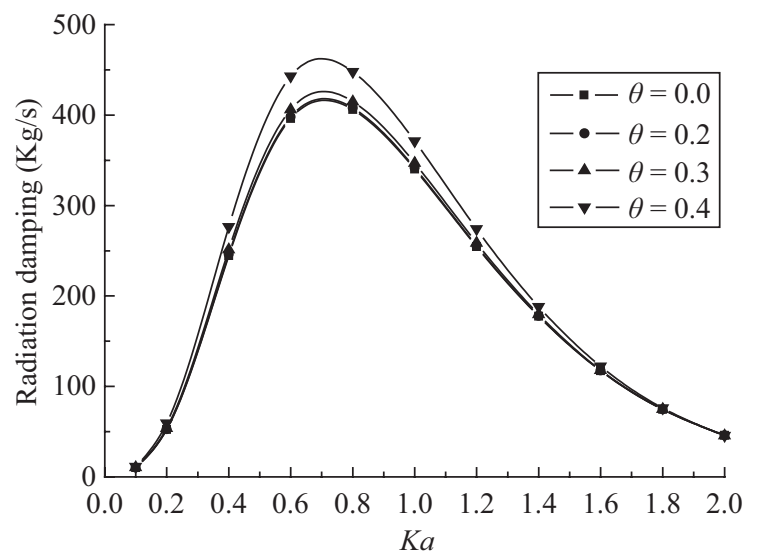

Fig. 3. Heave radiation damping for a submerged sphere with an opening hole.

different only when $K a$ is between 0.4 and 1.4. It is easy to draw a conclusion that heave added mass varies more obviously with opening hole angle increasing than radiation damping.

\section{CONCLUSIONS}

In the present study, an analytical method is developed to solve the radiation problem for a sphere with an opening hole in finite depth. Using multipole expansions, the fluid velocity potential has been obtained in the form of a double series of the associated Legendre functions. Subsequently, we analyze the added mass and radiation damping due to heave motions by the method of truncation. When angles of opening hole are increasing added mass tend to go up more obviously than radiation damping.

In the framework of linear potential theory, the proposed solution in this paper can be applied to optimization analysis for nonholonomic spherical structures in ocean engineering. Besides, this work may serve as a benchmark to check different numerical schemes for validation. 


\section{REFERENCES}

1. Abramowitz, M. and Stegun, I. A., Handbook of Mathematical Functions, Dover, New York (1965).

2. Bai, W. and Eatock Taylor, R., "Higher-order boundary element simulation of fully nonlinear wave radiation by oscillating vertical cylinders," Applied Ocean Research, Vol. 28, No. 4, pp. 247-265 (2006).

3. Datta, R. and Sen, D., "A B-spline-based method for radiation and diffraction problems," Ocean Engineering, Vol. 33, pp. 2240-2259 (2006).

4. Gao, Z. L. and Zou, Z. J., "A NURBS-based high-order panel method for three-dimensional radiation and diffraction problems with forward speed," Ocean Engineering, Vol. 35, pp. 1271-1282 (2008).

5. Gao, Z. L. and Zou, Z. J., "A three-dimensional desingularized high order panel method based on Nurbs," Journal of hydrodynamics, Vol. 20, No. 2, pp. 137-146 (2008).

6. Linton, C. M., "Radiation and diffraction of water waves by a submerged sphere in finite depth," Ocean Engineering, Vol. 18, No. 1, pp. 61-74 (1991).

7. Qiu, W. and Hsiung, C. C., "A panel-free method for time-domain analysis of the radiation problem," Ocean Engineering, Vol. 29, No. 12, pp. 1555-1567 (2002).

8. Rahman, M., "Simulation of diffraction of ocean waves by a submerged sphere in finite depth," Applied Ocean Research, Vol. 23, pp. 305-317
(2002).

9. Ratock Taylor, R. and Hu, C. S., "Multipole expansions for wave diffraction and radiation in deep water," Ocean Engineering, Vol. 18, No. 3, pp. 191-224 (1991)

10. Siddorn, P. and Eatock Taylor, R., "Diffraction and independent radiation by an array of floating cylinders," Ocean Engineering, Vol. 35, No. 13, pp. 1289-1303 (2008).

11. Teng, B. and Eatock Taylor, R., "New higher-order boundary element methods for wave diffraction/radiation," Applied Ocean Research, Vol. 17, No. 2, pp. 71-77 (1995).

12. Thorne, R. C., "Multipole expansions in the theory of surface waves," Proceedings of Cambridge Philosophical Society, Vol. 49, pp. 707-716 (1953).

13. Wang, S., "Motion of a spherical submarine in waves," Ocean Engineering, Vol. 13, No. 3, pp. 249-271 (1986).

14. Wang, Y. X. and Lu, X. Y., "Finite element analysis of viscous flow past a rotating sphere," Journal of Hydrodynamics, Ser. B, Vol. 13, No. 2, pp. 83-88 (2001).

15. Zheng, Y. H., Shen, Y. M., and Tang, J., "Radiation and diffraction of linear water waves by an infinitely long submerged rectangular structure parallel to a vertical wall," Ocean Engineering, Vol. 34, No. 1, pp. 69-82 (2007). 